\title{
RHIC data and small $x$ physics
}

\author{
T. Lappi ${ }^{\mathrm{a}} \mathrm{b} *$

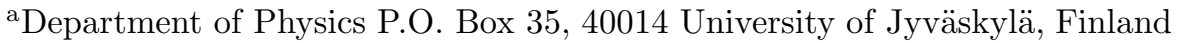 \\ bInstitut de Physique Théorique, Bât. 774, CEA/DSM/Saclay, 91191 Gif-sur-Yvette Cedex, France
}

This is a short review of some RHIC results that have been most important for the small $x$ physics community. We discuss saturation effects in deuteron-gold collisions, particle production in gold-gold collisions and some effects of the large "glasma" field configurations in the early stages of the collision.

\section{RHIC experiments}

The Relativistic Heavy Ion collider (RHIC) at Brookhaven National Laboratory (BNL) (see e.g. Ref. 11) has been colliding gold and lighter nuclei at an energy of $\sqrt{s}=200 A \mathrm{GeV}$ since 2000. The accelerator also delivers polarized proton beams at $\sqrt{s}=500 \mathrm{GeV}$, used to study spin physics. The main goal of the experimental program is the discovery and study of the Quark Gluon Plasma (see e.g. Refs. 2] for overall reviews of the experimental results).

There have been four major RHIC experiments, the two smaller ones now decommissioned and the two larger ones being upgraded and taking more data:

PHOBOS is a small detector, mostly of silicon. It has a huge acceptance in pseudorapidity $\eta$ and azimuthal angle $\phi$, but mostly without $p_{T}$ measurement or particle identification.

BRAHMS is also a small detector, consisting of 2 moveable spectrometer arms that give it a large $\eta$ coverage with particle identification, but a small acceptance.

PHENIX is a large detector optimized more for electromagnetic signals (photons, leptons) with a good acceptance but not full azimuthal coverage $(\Delta \phi=\pi$ in two spectrometer arms).

*Supported by the Academy of Finland, contract 126604.
STAR resembles most closely a typical high energy experiment, being built around a large TPC covering the full azimuthal angle. It is more optimized towards hadronic and global observables.

The near term future of the BNL program is to continue taking data and improving the PHENIX and STAR detectors in parallel to the LHC heavy ion program. In the longer term there are plans to build an Electron-Ion Collider (EIC) to collide electrons with high energy protons and nuclei [3]. At CERN the LHC will, in addition to the proton beams, have lead-lead (and eventually other species) collisions at $\sqrt{s}=5500 \mathrm{~A} \mathrm{GeV}$. There is one detector (ALICE) dedicated to heavy ions and smaller heavy ion programs in the CMS and ATLAS experiments.

An important tool for understanding the geometry of the collision goes by the name of Glauber modeling. Experimental data are usually presented divided into centrality bins determined mostly by the total charged multiplicity. A Glauber model treats the nucleus as a collection of loosely bound nucleons distributed according to the charge density distribution. These nucleons then scatter independently with the experimentally measured proton-proton cross section. A Glauber model the associates each impact parameter with a certain number of participant $n u-$ cleons $N_{\text {part }}$ and binary collisions $N_{\text {bin }}$. One thus obtains an estimate for the typical impact parameter corresponding to the centrality bins. While the assumption of independent collisions is not a 


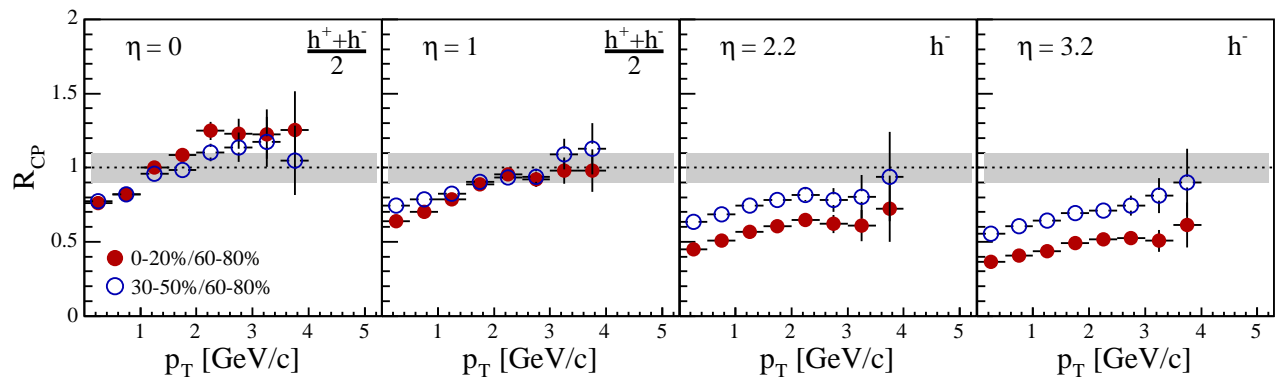

Figure 1. BRAHMS results on suppression of charged hadron production in the deuteron fragmentation region [4. The plot shows $R_{C P}$ (see text) for charged hadron spectra between two central bins (0-20\% and $30-50 \%)$ and peripheral collisions (60-80\% most central).

very realistic one at high energies, Glauber modeling has turned out to be a convenient way to parametrize the geometrical aspects of the collision; experimental data are usually expressed in terms of $N_{\text {part }}$ and $N_{\text {bin }}$. Experimental results for nuclear modifications to the spectra of produced particles are often presented as ratios (such as $R_{A A}$, ratio of nucleus-nucleus to proton-proton; $R_{d A u}$, deuteron-gold to proton-proton and $R_{C P}$, central to peripheral) corrected by the geometrical factor deduced from a Glauber calculation. These ratios are defined in such a way that in the case of independent scatterings of the nucleons in the nucleus they should be equal to one.

We can understand the time evolution of the collision process in different stages:

1. The initial condition at $\tau=0$ depends on the properties of the nuclear wavefunction at small $x$.

2. Thermal and chemical equilibration.

3. The Quark Gluon Plasma, surviving for some fermis around $\tau_{0} \lesssim \tau \lesssim 10 \mathrm{fm}$. If the system reaches local thermal equilibrium, finite temperature field theory and relativistic hydrodynamics can be used to describe its behaviour.

4. Finally, for $\tau \gtrsim 10 \mathrm{fm}$ the system hadronises and decouples.

In the remainder of this talk we will concentrate on the physics of the first stage, the initial condition.

\section{Color glass and glasma}

The central rapidity region in high energy collisions originates from the interaction of the "slow" small $x$ degrees of freedom, predominantly gluons, in the wavefunctions of the incoming hadrons or nuclei. At large energies these gluons form a dense system that is characterized by a transverse momentum scame $Q_{\mathrm{s}}$, the saturation scale. The degrees of freedom with $p_{T} \lesssim Q_{\mathrm{s}}$ are fully nonlinear Yang-Mills fields with large field strength $A_{\mu} \sim 1 / g$ and occupation numbers $\sim 1 / \alpha_{\mathrm{s}}$, they can therefore be understood as classical fields radiated from the large $x$ partons. Note that while this description is inherently nonperturbative, it is still based on weak coupling, because the classical approximation requires $1 / \alpha_{\mathrm{s}}$ to be large and therefore $Q_{\mathrm{s}} \gg \Lambda_{\mathrm{QCD}}$. A "pocket formula" for estimating the energy and nuclear dependence of the saturation scale is $Q_{\mathrm{s}}{ }^{2} \sim A^{1 / 3} x^{-0.3}$ : nonlinear high gluon density effects are enhanced by going to small $x$ and large nuclei. Ideally one would like to study the physics of the CGC at the Electron Ion Collider [3], but already based on fits to HERA data and simple nuclear geometry we have a relatively good idea of the magnitude of $Q_{\mathrm{s}}$ at RHIC energies [6. The CGC is a systematic effective theory (effective because the large $x$ part of the wavefunction is integrated out) formulation of these degrees of freedom. The term 

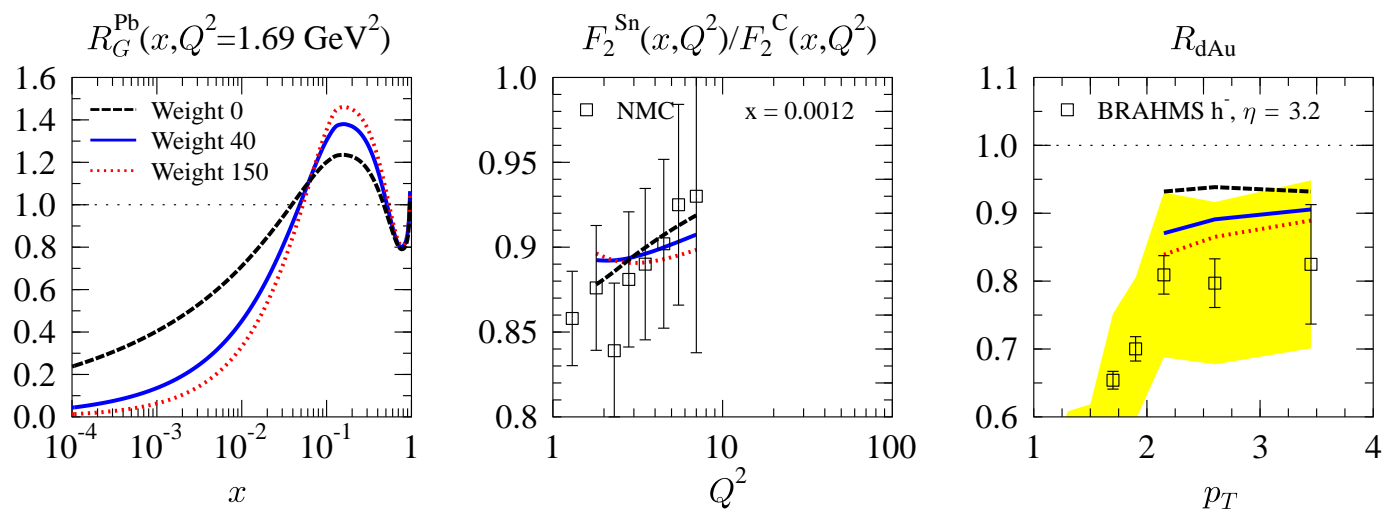

Figure 2. DGLAP parametrizations of nuclear pdf's from Ref. [5]. The left panel shows the suppression of the nuclear gluon distribution required to simultaneously fit the nuclear DIS data from NMC (center panel) and charged hadron production data from BRAHMS (right panel) and PHENIX data.

glasma 7 refers to the coherent, classical field configuration resulting from the collision of two such objects CGC.

\section{Deuteron-nucleus collisions}

A theoretically more controlled way to study the small- $x$ nuclear wavefunction is using protonnucleus (in practice deuteron-nucleus) collisions, especially at forward rapidity, i.e. in the fragmentation region of the proton. In this case final state interactions in the plasma phase are expected to be absent, and the relevant degrees of freedom in the proton or deuteron are the relatively well understood large $x$ parton distributions.

The effects of parton saturation are particularly visible in the BRAHMS data (Fig. 1) for charged hadron production, which shows a significant depletion towards the deuteron fragmentation region. Although these data DGLAP-based 5 analysis can reproduce this data, see Fig. 2, this requires that the gluon distribution at small $x$ is suppressed by a factor of $\sim 10$ compared to the proton. This is a clear signal of the breakdown of the DGLAP picture and the importance of saturation effects. The suppression pattern at large rapidities was predicted from the CGC 8 and later more detailed calculations have quite successfully reproduced it, see Fig. 3.

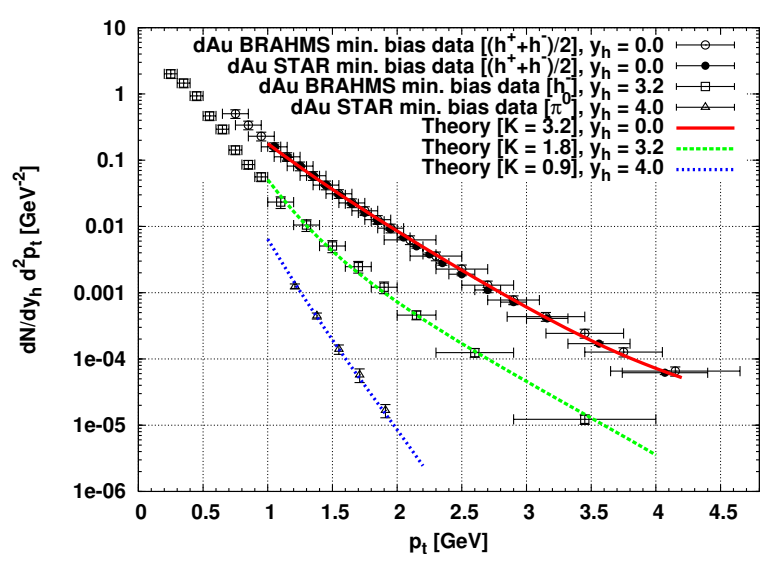

Figure 3. CGC calculation of charged hadron production 9 .

\section{Particle production and geometry}

In order to compute particle production in the Glasma one is presented with the following situation [10]. The valence-like degrees of freedom of the two nuclei are represented by two classical color currents that are, because of their large longitudinal momenta $\left(p^{ \pm}\right)$well localized on the light cone (in the variables conjugate to $p^{ \pm}$, namely $\left.x^{\mp}\right): J^{ \pm} \sim \delta\left(x^{\mp}\right)$. These then generate the classical field that one wants to find. Working in light cone gauge the field in the region of space- 


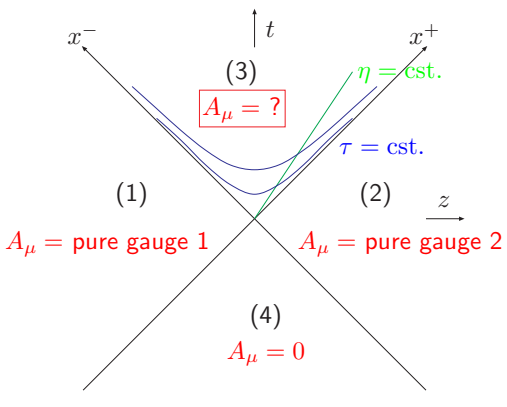

Figure 4. Classical field configurations.

time causally connected to only one of the nuclei (regions (1) and (2) in Fig. (4) is a transverse pure gauge, independently for each of the two nuclei. These pure gauge fields then give the initial condition on the future light cone $\left(\tau=\sqrt{2 x^{+} x^{-}}=0\right)$ for the nontrivial gauge field after the collision (region (3) in Fig. 4). The field inside the future light cone can then be computed either numerically [11] or analytically in different approximations (see e.g. 12 for recent work). The obtained result is then averaged over the configurations of the sources $J^{\mu}$ with a distribution $W_{y}\left[J^{\mu}\right]$ that includes the nonperturbative knowledge of the large $x$ degrees of freedom. The resulting fields are then decomposed into Fourier modes to get the gluon spectrum. This is the method that we will refer to as Classical Yang-Mills (CYM) calculations.

In the limit when either one or both of the color sources are dilute (the "pp" and "pA" cases), the CYM calculation can be done analytically and reduces to a factorized form in terms of a convolution of unintegrated parton distributions that can include saturation effects. Although this approach (often known as "KLN" after the authors of [13]) is not is not derived for the collision of two dense systems, it has advantage of offering more analytical insight and making it easier to incorporate large- $x$ ingredients into the calculation.

The CYM calculations [11] of gluon production paint a fairly consistent picture of gluon production at RHIC. The estimated value $Q_{\mathrm{s}} \approx 1.2 \mathrm{GeV}$ from HERA data [6] (corresponding to the MV model parameter $g^{2} \mu \approx 2.1 \mathrm{GeV}$ [15]) leads to

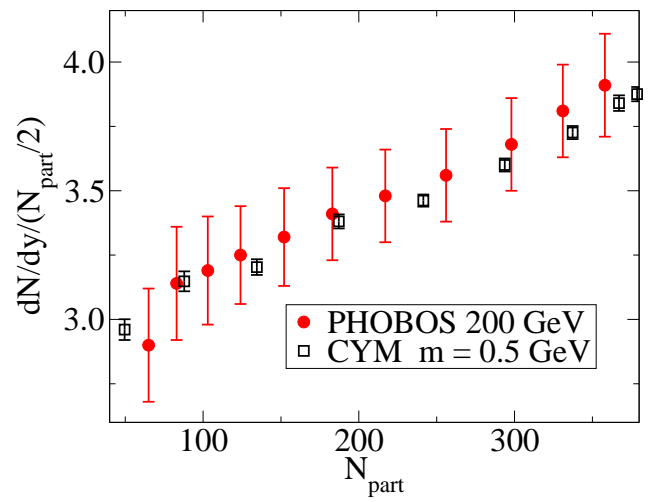

Figure 5. Centrality dependence of the multiplicity from a CYM calculation [14].

$\frac{\mathrm{d} N}{\mathrm{~d} y} \approx 1100$ gluons in the initial stage. Assuming a rapid thermalization and nearly ideal hydrodynamical evolution this is consistent with the observed $\sim 700$ charged $(\sim 1100$ total $)$ particles produced in a unit of rapidity in central collisions.

Of the more detailed geometrical aspects of the initial condition the basic features (such as the closeness to $N_{\text {part }}$ scaling) of the centrality dependence of particle multiplicities are mostly straightforward consequences of the proportionality of the multiplicity to $Q_{\mathrm{s}}{ }^{2}$; they are successfully reproduced by both KLN and CYM calculations [131416], see Fig. [5.

A striking signal of collective behavior of the matter produced at RHIC is elliptic flow; where the initial spatial anisotropy in a noncentral collision is transformed to an anisotropic distribution of produced particles in momentum space through collective flow caused by the pressure gradients. Comparing hydrodynamical calculations with measurements is a way to address fundamental properties of the medium, such the equation of state or viscosity, but this requires understanding of the initial conditions. These initial conditions have traditionally been phenomenologically parametrized in terms of the number of nucleons participating in the collision. The CGC provides a method to compute them from first principles, and has changed the interpretation of the experiments in terms of the viscosity [14 17]. 

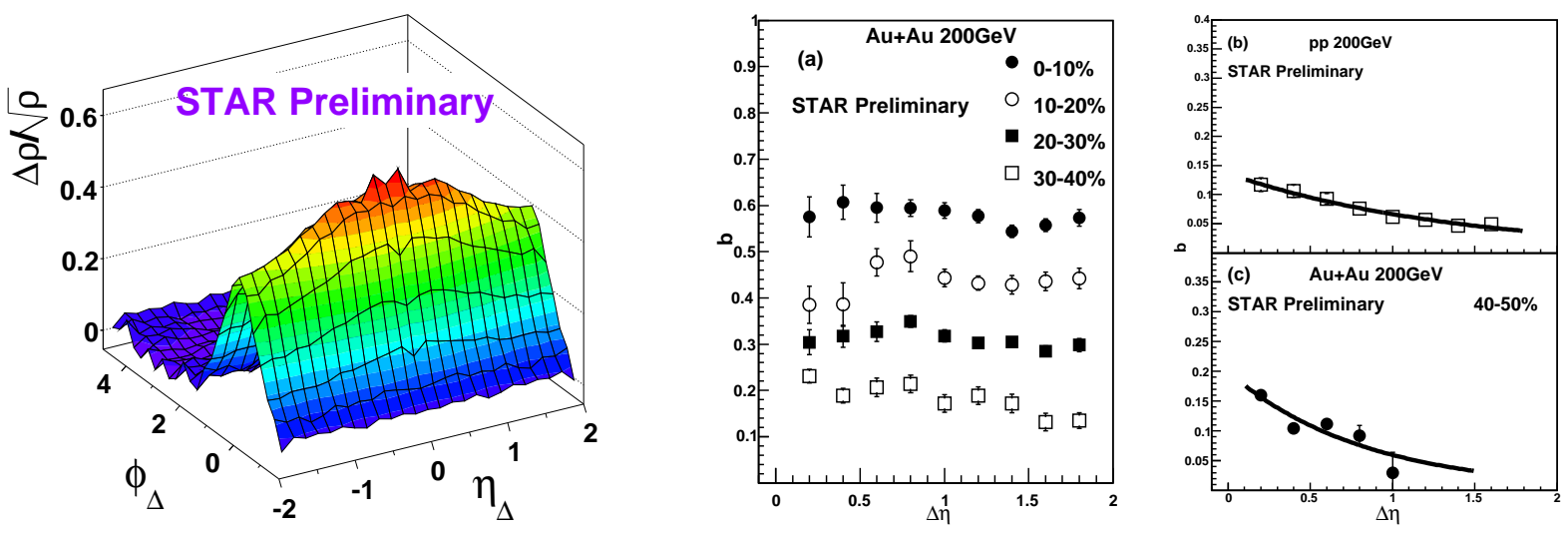

Figure 6. Left: Two particle correlation measurement from STAR, showing the "ridge" structure elongated in pseudorapidity. Right: Long range correlations in multiplicity: $b=\frac{\left\langle N_{F} N_{B}\right\rangle-\left\langle N_{F}\right\rangle\left\langle N_{B}\right\rangle}{\left\langle N_{F}^{2}\right\rangle-\left\langle N_{F}\right\rangle^{2}}$, where $F$ and $B$ are pseudorapidity bins separated by $\Delta \eta$.

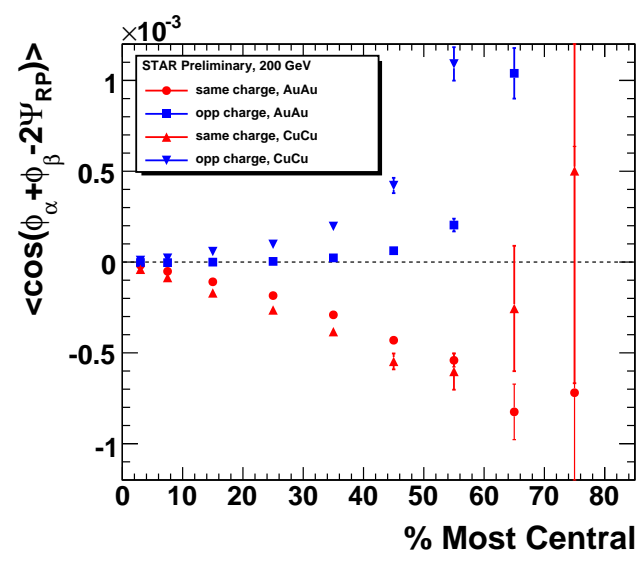

Figure 7. Parity violating correlation between reaction plane and momentum [19].

An example of a more exclusive probe of the medium is the $J / \Psi$. It has been called the "thermometer of the QGP", because it the state is expected from lattice calculations to melt at a temperature close to the QCD phase transition. The production mechanism of the $J / \Psi$ from perturbative QCD is not very well understood, and since the charm quark mass is close to the typical values $Q_{\mathrm{s}}$, it can be expected to be very sensitive to saturation physics [18].

\section{Glasma physics}

Correlations over large distances in rapidity can, by causality, only originate from the earliest times in the collision process, and are thus sensitive to the properties of the glasma phase of the collision. Examples of such phenomena are the elongated "ridge" structure seen in two particle correlations and long range correlations in multiplicity (see Fig. 6) [20]. The boost invariant nature of the Glasma fields naturally leads to this kind of a correlation, and deviations from it should be calculable from the high energy evolution governing the rapidity dependence [21].

Another remarkable phenomenon that is possible in the Glasma field configuration is the generation of a large Chern-Simons charge and thus parity violation 22] due to the nonperturbatively large field configurations. Through the so called "chiral magnetic effect" this can manifest itself in a parity violating correlation between the electric dipole moment (or momentum anisotropy between negative and positive charges; a vector) and the reaction plane (the positive charges of the ions generate a magnetic field perpendicular to the reaction plane; a pseudovector). There are some preliminary indications in the data of a nonzero value for such an observable [19].

In conclusion, experiments at RHIC have 
clearly produced a strongly interacting deconfined form of matter, but the full understanding of the experimental results requires an understanding of the small $x$ physics giving the initial conditions of the collision process. This is the domain of strongly nonliear gluon fields at high energy, best understood in terms gluon saturation and the Color Glass Condensate effective theory. This framework provides a way to calculate properties of the initial condition from first principles, relate them to DIS measurements, and understand some of the observables that directly probe the initial condition. With the much greater collision energy of the LHC these effects are likely to become even more prominent.

\section{REFERENCES}

1. J. L. Nagle and T. S. Ullrich, arXiv:nucl-ex/0203007

2. STAR, J. Adams et al., Nucl. Phys. A757, 102 (2005), arXiv:nucl-ex/0501009; PHENIX, K. Adcox et al., Nucl. Phys. A757, 184 (2005), arXiv:nucl-ex/0410003; BRAHMS, I. Arsene et al., Nucl. Phys. A757, 1 (2005), arXiv:nucl-ex/0410020; B. B. Back et al., Nucl. Phys. A757, 28 (2005), arXiv:nucl-ex/0410022.

3. A. Deshpande, R. Milner, R. Venugopalan and W. Vogelsang, Ann. Rev. Nucl. Part. Sci. 55, 165 (2005), arXiv:hep-ph/0506148.

4. BRAHMS, I. Arsene et al., Phys. Rev. Lett. 93, 242303 (2004), arXiv:nucl-ex/0403005.

5. K. J. Eskola, H. Paukkunen and C. A. Salgado, JHEP 07, 102 (2008), arXiv:0802.0139 [hep-ph].

6. H. Kowalski, T. Lappi and R. Venugopalan, Phys. Rev. Lett. 100, 022303 (2008), arXiv:0705.3047 [hep-ph]].

7. T. Lappi and L. McLerran, Nucl. Phys. A772, 200 (2006), arXiv:hep-ph/0602189.

8. D. Kharzeev, Y. V. Kovchegov and K. Tuchin, Phys. Rev. D68, 094013 (2003), arXiv:hep-ph/0307037.

9. A. Dumitru, A. Hayashigaki and J. JalilianMarian, Nucl. Phys. A770, 57 (2006), arXiv:hep-ph/0512129.

10. A. Kovner, L. D. McLerran and
H. Weigert, Phys. Rev. D52, 3809 (1995), arXiv:hep-ph/9505320.

11. A. Krasnitz, Y. Nara and R. Venugopalan, Phys. Rev. Lett. 87, 192302 (2001), arXiv:hep-ph/0108092; T. Lappi, Phys. Rev. C67, 054903 (2003), arXiv:hep-ph/0303076; A. Krasnitz, Y. Nara and R. Venugopalan, Nucl. Phys. A727, 427 (2003), arXiv:hep-ph/0305112.

12. J.-P. Blaizot and Y. Mehtar-Tani, Nucl. Phys. A8018, $97 \quad$ (2009), arXiv:0806.1422 [hep-ph].

13. D. Kharzeev and M. Nardi, Phys. Lett. B507, 121 (2001), arXiv:nucl-th/0012025; D. Kharzeev and E. Levin, Phys. Lett. B523, 79 (2001), arXiv:nucl-th/0108006.

14. T. Lappi and R. Venugopalan, Phys. Rev. C74, 054905 (2006), arXiv:nucl-th/0609021.

15. T. Lappi, Eur. Phys. J. C55, 285 (2008), arXiv:0711.3039 [hep-ph]].

16. H.-J. Drescher and Y. Nara, Phys. Rev. C76, 041903 (2007), arXiv:0707.0249 [nucl-th]].

17. T. Hirano, U. W. Heinz, D. Kharzeev, R. Lacey and Y. Nara, Phys. Lett. B636, 299 (2006), arXiv:nucl-th/0511046; H. J. Drescher and Y. Nara, Phys. Rev. C75, 034905 (2007), arXiv:nucl-th/0611017.

18. D. Kharzeev, E. Levin, M. Nardi and K. Tuchin, arXiv:0808.2954 [hep-ph],

19. STAR, S. A. Voloshin, arXiv:0806.0029 [nucl-ex],

20. J. Putschke, J. Phys. G34, S679 (2007), arXiv:nucl-ex/0701074; STAR, M. Daugherity, arXiv:0806.2121 [nucl-ex], STAR, B. K. Srivastava, Int. J. Mod. Phys. E16, 3371 (2008), arXiv:nucl-ex/0702054.

21. A. Dumitru, F. Gelis, L. McLerran and R. Venugopalan, Nucl. Phys. A810, 91 (2008), arXiv:0804.3858 [hep-ph]; F. Gelis, T. Lappi and R. Venugopalan, arXiv:0810.4829 [hep-ph],

22. D. Kharzeev, A. Krasnitz and R. Venugopalan, Phys. Lett. B545, 298 (2002), arXiv:hep-ph/0109253; D. E. Kharzeev, L. D. McLerran and H. J. Warringa, Nucl. Phys. A803, 227 (2008), arXiv:0711.0950 [hep-ph]. 